\title{
Dermatological Manifestations of COVID-19: A Brief Review
}

\author{
Harsimran Kaur ${ }^{1}$ \\ ${ }^{1}$ Department of Dermatology, Guru Harkrishan Hospital, New Delhi, \\ India
}

Address for correspondence Harsimran Kaur, MBBS, MD, MRCP, 57-A, Pocket-C, Siddhartha Extension, New Delhi 110014, India (e-mail: harsimran688@yahoo.com).

\begin{abstract}
Keywords

- COVID-19

- cutaneous manifestations

- SARS-CoV2

- teledermatology

- viral mimicker

The emphasis so far during the COVID-19 pandemic has been on the respiratory manifestations with little attention being paid to cutaneous manifestations. The novel coronavirus has a wide spectrum of cutaneous manifestations which are broadly divided into exanthematous and vasculopathic type of lesions. The effects of this novel virus on the integumentary system cannot be underestimated as it can mimic various types of viral skin lesions. Thus, dermatologists should have knowledge about COVID-19 disease presentations and which differential diagnosis to consider if they encounter skin lesions in a patient who is known or suspected to have COVID-19. Patient evaluation and workup also needs to be modified during the time of this pandemic. Immunosuppressive/immunomodulatory drugs which are rampantly used in dermatological practice, must be used only after weighing their risks and benefits during the COVID-19 era. Personal Protective Equipment has to be worn when coming in contact with a suspected or a proven case of COVID-19. However, its use itself is associated with dermatological side effects which should be known to dermatologist practising during the COVID-19 era. Teledermatology can go a long way in circumventing these issues and it should be made more accessible, especially in remote areas. Another future recommendation could be setting up of a national level organization or group for recording dermatological data related to COVID-19.
\end{abstract}

\section{The COVID-19 Pandemic: Problem Statement}

After the first report of an atypical case of pneumonia from Wuhan, China, in December 2019, novel coronavirus disease 2019 (COVID-19) caused by the severe acute respiratory syndrome-coronavirus-2 (SARS-CoV-2) has spread like wildfire across all continents. It was declared as a global pandemic by the World Health Organization (WHO) on March 11, 2020. At the time of writing this article, COVID-19 has already affected 5.1 million individuals and caused more than 329,895 deaths worldwide. In India, there are 1.12 lakh individuals who have been infected and 3,435 deaths so far. In terms of the number of cases, the worst affected Indian states are Maharashtra, Tamil Nadu, Gujarat, Delhi, Rajasthan, Madhya Pradesh, Uttar Pradesh, Andhra Pradesh, Bihar, and Punjab respectively, whereas other states have reported fewer cases. The emphasis so far, has predominantly been on the respiratory manifestations of

published online

September 17, 2020
DOI https://doi.org/

10.1055/s-0040-1716938 ISSN 0379-038X. this illness and only little is known about the various dermatological manifestations in COVID-19. Though there are multiple case reports from around the world on the direct and indirect cutaneous manifestations associated with this pandemic, literature from India is scant. While an initial study in China reported the incidence of cutaneous rash to be $0.2 \%$ among COVID-19 cases, it was subsequently found to be $20.4 \%$ in an Italian study. ${ }^{1,2}$ Thereafter, manifestations have been reported in asymptomatic carriers or healthy contacts of affected cases, thus emphasizing the fact that the effect of the said viral infection cannot be underestimated on the integumentary system.

\section{Cutaneous Manifestations of COVID-19: The Evidence so Far}

Multiple patterns of dermatological lesions have been reported among patients infected by the SARS-CoV-2. The first 
study on cutaneous manifestations of the COVID-19 pandemic reported erythematous rash in 14 out of 18 patients, urticaria in 3 , and vesicular rash in 1 patient. $^{2}$ Thereafter, there have been a multitude of isolated case reports and case series. As suggested by Suchonwanit et al, skin lesions in COVID-19 may be broadly classified into two categories, that is, exanthematous or vasculopathic type of lesions. ${ }^{3}$

Among the vasculopathic type, acroischemic lesions have been the most frequently reported among COVID-19 patients. These lesions have been nicknamed as "COVID toes" by the print and electronic media. They characteristically affect healthy children or adolescents who develop multifocal, asymmetric erythematous, or erythemoviolaceous round lesions up to few millimeters in size on several toes with unaffected interposed areas and clear metatarsophalangeal delineation. These lesions characteristically evolve from erythema, ecchymosis to vesiculobullous forms, and ultimately to a superficial necrosis stage (blackish crusting) over the next few days. Itching, burning, joint movement restriction, and pain are the most frequently reported symptoms. ${ }^{4}$ Acroischemic lesions were grouped as chilblain like lesions in 95 out of 132 cases (72\%) and erythema multiforme (EM) like in remaining $28 \%$ cases in a large retrospective Spanish case series. ${ }^{5}$ These lesions are quite specific among those with a family history or travel history of exposure and thus may be a cutaneous marker in such suspected COVID-19 cases. ${ }^{5}$ Such lesions usually appear after a few days of mild respiratory symptoms (mean latency of 9.2 days in the Spanish series) or in asymptomatic cases, hence their presence raises questions about their clinical implication. The mean duration of these self-resolving lesions was 8.2 days. Other patterns of acroischemic lesions include EM-like lesions which were found in $28 \%$ of patients of the acroischemic cohort. These too present on tips of fingers and toes, as small as $(<1 \mathrm{~cm})$ coalescent round erythematous macules and vesicles. Unlike classical EM, they are localized and don't have a targetoid morphology. ${ }^{5}$ Feet were the most commonly involved site $(81.8 \%)$ in cases of both chilblain and EM-like lesions followed by both hands and feet and hands alone. EM lesions were found to involve elbows, knees, and ears in only $5.4 \%$ of the cases. All of the above findings were consistent in a large scale online study conducted in 63 patients with preliminary acral lesions in Italy as well. ${ }^{6}$

Violaceous macules with porcelain-like appearance, eruptive cherry angiomas, and chilblains with Raynaud's phenomenon were some other rare vasculopathic variants reported in a French study in swab-positive COVID-19 patients. $^{7}$ There have also been reports of true ischemic lesions which resemble gangrene in severely affected COVID-19 pneumonia patients with evidence of disseminated intravascular coagulation (DIC). Acroischemic lesions in such severely affected patients are usually associated with thrombocytopenia, elevated D-Dimer levels, and altered prothrombin time. ${ }^{8}$ Anti phospholipid antibodies were also noted in one such patient. ${ }^{9}$
Manalo et al have reported two cases of late onset evanescent, nonpruritic, lacy, erythematous, blanching livedo reticularis-like lesions in nasopharyngeal swab polymerase chain reaction (PCR)-confirmed COVID-19 cases mainly affecting the thigh area. ${ }^{10}$ Another interesting case was reported, wherein a symptomatic SARS-CoV-2-positive patient developed erythematous yellowish papules on both heels on day 13 after being tested positive. These lesions then evolved into hard pruritic plaques 3 days later. Though the patient could not be biopsied, clinical differentials included urticarial vasculitis, plantar hidradenitis, or neutrophilic dermatosis. ${ }^{11}$

There have been case reports of acute onset urticaria with concomitant pyrexia as inaugural symptoms of SARS$\mathrm{CoV}-2$ infection which were later found positive on nasopharyngeal swab PCR. All these patients typically lacked lung involvement, while associated anosmia, ageusia, and odynophagia were more common. Also, they were easy to treat with second generation antihistaminics and paracetamol. ${ }^{12-14}$

Regarding exanthematous lesions, a varicella-like exanthem was reported in a multicenter case series of 22 COVID-19-positive patients. Median onset of lesions was 3 days after symptom onset with subsequent resolution over 8 days. Histopathology was suggestive of basal layer vacuolization with hyperchromatic multinucleate keratinocytes. However, unlike classic varicella, these lesions were monomorphic, predominantly involved the trunk, with mild or no pruritus, and resolved without scarring. ${ }^{15}$ There have been anecdotal reports of a few patients with herpetiform lesions as well, but it is unclear, whether herpes zoster in immunosuppressed COVID-19 affected patients or a manifestation of the COVID-19 disease itself. ${ }^{16}$

In addition to the morbiliform maculopapular viral rash, ${ }^{17}$ a few peculiar exanthematous patterns have also been reported. Joob and Wiwanitkit reported a petechial rash which was misdiagnosed as dengue rashes prior to a reverse transcript (RT)-PCR that suggested corona virus infection..$^{18}$ There are two reports in literature of COVID-19positive patients presenting as a periflexural exanthem. Symmetrical drug-related intertriginous and flexural exanthema (SDRIFE) like exanthematous rash maybe caused classically by a drug or rarely in a parvoviral infection; however, in a reported case, the rash disappeared despite continuation of the drug, thus emphasizing the need to consider corona virus infection as a possible etiology. ${ }^{19,20}$ Some reports have described an annular, circinate, edematous, fixed, nonpruritic plaque-type febrile rash in COVID-19-inflicted cases. The rash was likened to pityriasis rosea (PR) in one of these reports. Both the patients had mild symptoms and a favorable outcome. ${ }^{21,22}$ Thereafter, an atypical digitate papulosquamous variant of PR has been reported by Sanchez et al, wherein the clinical findings were confirmed on histopathology. ${ }^{23}$ A 6-month-old infant with classic Kawasaki's disease, having an erythematous blotchy rash, prominent tongue papilla, and cracked lips as her predominant mucocutaneous features, was surprisingly found to be COVID-19-positive through RT-PCR test. ${ }^{24} \mathrm{~A}$ diffuse maculopapular and urticarial 
rash has also been reported in a COVID-19 positive lady who developed rash on her postpartum day $10 .{ }^{25}$

\section{Indirect Cutaneous Associations of COVID-19 Pandemic}

Goren et al have made an observational hypothesis of increased prevalence of SARS-CoV-2 infection in males suffering from male pattern hair loss (MPHL). This is attributed to the fact that human androgens are the only known transcription promoters of TMPRSS2 gene that helps in proteolytic priming of the COVID-19 spike glycoprotein. This step is essential in subsequent binding of the virus to host angiotensin-converting enzyme 2 (ACE2) receptor and facilitates viral cell entry. Thus, there may be an association as to why COVID-19 is particularly affecting males with MPHL. ${ }^{26}$

There has been an increase in the incidence of occupational contact dermatitis in health care workers using hand gloves and personal protective equipment (PPE). Adverse reactions were more common on continuous use of PPE for more than 6 hours and with the use of respirators instead of masks. Hands, cheek, and nasal bridge were the most affected areas and dryness or scaling, papules, erythema, and maceration were reported as the most common cutaneous findings in a multicenter cross-sectional study conducted in Wuhan, China. ${ }^{27}$ The general increase in incidence of acne and miliaria rubra and pustulosa has been mentioned in literature with PPE use; however, there are no proper studies as yet to confirm the same.

A rising trend in prevalence of hand eczema has been observed in an Indian study conducted in Nagpur due to overzealous hand hygiene practices leading to impairment of skin barrier. ${ }^{28}$

\section{Plausible Pathogenic Mechanisms of Various Dermatological Manifestations}

An extensive study was performed to delineate the pathogenic mechanism behind the cutaneous manifestations in COVID-19 affected patients. A pauci-inflammatory thrombogenic vasculopathy, with deposition of C5b-9 and C4d both involved, and normal appearing skin was identified in this study. This signifies immune complex-mediated activation of alternate and lectin complement pathways leading to microvascular thrombosis and associated vasculopathic cutaneous lesions. ${ }^{29}$ Acroischemia in critically ill COVID-19 patients has been associated with a possible hypercoaguable state with raised D-dimer levels and a deranged prothrombin time. ${ }^{8}$ However, majority of studies have reported an outbreak of perniotic skin lesions in asymptomatic healthy or mildly symptomatic adolescents or young adults who may have come in contact with a COVID19 case or an exposed health care worker. The mechanism in such patients may be complement and immune complexmediated microvascular thrombosis. Very often, these cases were found to be swab negative which has been attributed to the fact that these lesions are a very late manifestations when the viral load is low and PCR may have already reversed to negative. Another hypothesis for PCR negativity in such lesions could be an immunologically delayed response in genetically predisposed individuals. These cases contribute to a large proportion of viral reservoir and the onus of trying to identifying or catching them early lies solely on the dermatologist.

Maculopapular rash in viral exanthems may be explained by vasculitic changes in the small superficial blood vessels. For vesicular exanthems associated with COVID-19 patients, one may hypothesize that lymphocyte-associated viremia provides the virus access to epidermal keratinocytes. Viral replication in these cells with subsequent fusion leads to multinucleate cell formation and ballooning degeneration resulting in vesiculation.

The association between urticaria and viral fevers is well known. It may be postulated that cross reaction between COVID-19 viral antibodies and mast cell immunoglobulin (Ig)-E causes mast cell degranulation with release of proinflammatory mediators. Another plausible mechanism may be via circulating immune complexes which may stimulate basophils. This may further lead to release of vasoactive amines and complement activation. All of these mechanisms cause a transient increase in vascular permeability and result in urticarial lesions.

PR is known to be associated with reactivation of human herpes virus 6 and 7, Epstein-Barr virus, other viral infections, drugs, and vaccines. PR is usually an autoaggressive genetically mediated reaction pattern secondary to a viral trigger due to chemokine/cytokine release. Similar assumptions may be thus be drawn for a PR-type rash among COVID-19 patients.

Various studies have described different viral respiratory infections as a trigger or a sequelae to Kawasaki's disease. Some of these include enterovirus, parainfluenza, respiratory syncytial virus, influenza, adenovirus, and previous strains of human corona virus (strains 229E, HKU1, NL63, and OC43). ${ }^{24}$ Since prior human corona virus strains have been associated with the occurrence of Kawasaki's disease, SARS$\mathrm{CoV}-2$ may be a trigger for Kawasaki's disease as well.

\section{Patient Evaluation}

Apart from complete hemogram, liver, and kidney function tests, there is special emphasis on checking C-reactive protein levels, D-dimer assay, or fibrin degradation products (FDP) and prothrombin time. Elevated D-dimer and altered prothrombin time are considered poor prognostic markers..$^{30}$ COVID-19 status should be ascertained based on standardized RT-PCR kits and confirmed by serological tests for viral antibodies as per availability.

The pattern of rash should be evaluated and a detailed drug history should always be taken, as the closest mimicker of a viral exanthem is a drug rash. Dermatologist must be well aware of mucocutaneous side effects of all COVID-19 treatment drugs and a temporal relationship must be established between symptom onset, appearance of rash, associated changes with intake, or withdrawal of said drug. As the said pandemic is a great viral mimicker, other typical 
viral infections should be ruled out, preferably by serology, thus aiding in establishing a diagnosis by exclusion.

Histopathology should be undertaken with due precaution in all possible cases to establish diagnostic patterns. Apart from classic histopathology of viral exanthem, extremely dilated dermal blood vessels are the only reported diagnostic finding so far. ${ }^{31}$

It is essential to suspect COVID-19 infection during this pandemic time while evaluating acute onset febrile urticaria or cases of atypical vesiculation, especially in those who have had a history of varicella in the past and are already seroimmune.

Acroischemia must compel a dermatologist to take history of primary chilblains, Raynaud's autoimmune disorders, vasculitis, and coagulopathies in the past. Inciting factors must be probed into and an autoimmune antibody profile must be checked. Also, complete contact tracing must be done in cases of asymptomatic acroischemia if all of the above etiologies are ruled out. Asymptomatic acroischemic lesions may be regarded as a good prognostic marker as most of these patients did not develop frank COVID-19 symptoms.

\section{COVID-19 Pandemic and the Use of Immunosuppresants/Immunomodulators}

There is a question mark whether immunosuppressants/ immunomodulators or biologics should be continued in the COVID-19 era. Non biologic immunosuppressants, other than corticosteroids, are easier to stop and restart in a few days. However, biologics have a long half-life and they cannot be stopped abruptly due to the risk of antidrug antibody formation. Abrupt stoppage not only leads to disease worsening but can also cause a systemic cytokine storm which is shown to have a worse outcome in many COVID-19 patients. Hence, one must weigh the benefits versus the risk, before starting any patient on biologics or continuing biologics in patients with skin diseases.

Interdisciplinary risk assessment is a must before stopping immunosuppressives/immunomodulators for a patient who tests positive for COVID-19 infection. General guidelines so far suggest that interleukin inhibitors (secukinumab, dupilumab, anakinra, ustekinumab), phosphodiesterase-4 inhibitors (apremilast) and tumor necrosis factor (TNF)- $\alpha$ inhibitors (infliximab, adalimumab, and etanercept) can be continued unless viral symptoms are worsening or severe; however, corticosteroids should be tapered and antimetabolites (azathioprine, mycophenolate mofetil, and methotrexate), calcineuerin inhibitors (cyclosporine and tacrolimus), and anti CD-20 antibody (rituximab) should be stopped after symptom onset. ${ }^{32}$

\section{Future Implications}

To minimize direct contact, most cases should be seen virtually and in-person appointments should be set only if needed. Repeated use of disinfectants in clinics, appropriate use of PPE, and use of electronic prescriptions are some of the other essential measures in the times to come.
Digital photography through use of low density polythene covers and skin biopsy with the use of minimal instruments was suggested and tried in a hospital in Spain. ${ }^{33}$ Similar such guidelines and practices should be developed in the Indian scenario.

Dermoscopy should be avoided if possible and noncontact dermoscopy should be preferred.

Phototherapy chambers must be used judiciously (preferably for patients with high disease severity scores) with long gaps between patients and with specific cleaning protocols. ${ }^{34}$ Patients with mild or moderate disease severity should be deferred and maintained on topicals or systemic therapy.

The American Academy of Dermatology (AAD) COVID-19 Taskforce has developed an international registry to record cutaneous manifestation of COVID-19 cases and to keep a track of the effect of said pandemic on existing dermatological disorders. ${ }^{35}$ Dermatologists should be well aware of the facility and formation of similar registries at the national level can go a long way in data collection and disease pattern identification.

Since most dermatological conditions are chronic, doctors should encourage patients to join and interact in virtual support groups which may alleviate the psychological burdens of these patients. There should be awareness among dermatologists about these groups and the same information should be provided to patients in such testing times.

Also, we have the added responsibility to convey medical knowledge of right skin care and personal protective measures to the masses through social media.

\section{Conclusion}

COVID-19 pandemic has impacted our personal and professional lives to a great extent. We must continuously update our knowledge about its varied cutaneous manifestations and change our practice techniques with the changing times.

\section{Conflict of Interest}

None declared.

\section{References}

1 Guan WJ, Ni ZY, Hu Y, et al; China Medical Treatment Expert Group for Covid-19. Clinical characteristics of coronavirus disease 2019 in China. N Engl J Med 2020;382(18):1708-1720

2 Recalcati S. Cutaneous manifestations in COVID-19: a first perspective. J Eur Acad Dermatol Venereol 2020;34(5):e212-e213

3 Suchonwanit P, Leerunyakul K, Kositkuljorn C. Cutaneous manifestations in COVID-19: Lessons learned from current evidence. J Am Acad Dermatol 2020;83(1):e57-e60

4 Mazzotta F, Troccoli T. Acute acro-ischemia in the child at the time of COVID-19. Eur J Pediat Dermatol 2020;30(2):71-74

5 Fernandez-Nieto D, Jimenez-Cauhe J, Suarez-Valle A, et al. Comment on "Characterization of acute acro-ischemic lesions in non-hospitalized patients: a case series of 132 patients during the COVID-19 outbreak" J Am Acad Dermatol 2020;83(3):e241-e241 
6 Piccolo V, Neri I, Filippeschi C, et al. Chilblain-like lesions during COVID-19 epidemic: a preliminary study on 63 patients. J Eur Acad Venereol 2020;34(7):e291-e293

7 Bouaziz JD, Duong T, Jachiet M, et al. Vascular skin symptoms in COVID-19: a French observational study. J Eur Acad Dermatol Venereol 2020. Doi: 10.1111/jdv.16544

8 Zhang Y, Cao W, Xiao M, et al. [Clinical and coagulation characteristics of 7 patients with critical COVID-2019 pneumonia and acro-ischemia] (in Chinese). Zhonghua Xue Ye Xue Za Zhi 2020. Doi: 10.3760/cma.j.issn.0253-2727.2020.0006

9 Ma J, Xia P, Zhou Y, et al. Potential effect of blood purification therapy in reducing cytokine storm as a late complication of critically ill COVID-19. Clin Immunol 2020;214:108408

10 Manalo IF, Smith MK, Cheeley J, Jacobs R. A dermatologic manifestation of COVID-19: Transient livedo reticularis. J Am Acad Dermatol 2020;83(2):700

11 Estébanez A, Pérez-Santiago L, Silva E, Guillen-Climent S, García-Vázquez A, Ramón MD. Cutaneous manifestations in COVID-19: a new contribution. J Eur Acad Dermatol Venereol 2020;34(6):e250-e251

12 Henry D, Ackerman M, Sancelme E, Finon A, Esteve E. Urticarial eruption in COVID-19 infection. J Eur Acad Dermatol Venereol 2020;34(6):e244-e245

13 van Damme C, Berlingin E, Saussez S, Accaputo O. Acute urticaria with pyrexia as the first manifestations of a COVID-19 infection. J Eur Acad Dermatol Venereol 2020;34(7):e300-e301

14 Quintana-Castanedo L, Feito-Rodríguez M, Valero-López I, Chiloeches-Fernández C, Sendagorta-Cudós E, Herranz-Pinto P. Urticarial exanthem as early diagnostic clue for COVID-19 infection. JAAD Case Rep 2020;29(6):498-499

15 Marzano AV, Genovese G, Fabbrocini G, et al. Varicella-like exanthem as a specific COVID-19-associated skin manifestation: multicenter case series of 22 patients. J Am Acad Dermatol 2020;83(1):280-285

16 Tammaro A, Adebanjo GAR, Parisella FR, Pezzuto A, Rello J. Cutaneous manifestations in COVID-19: the experiences of Barcelona and Rome. J Eur Acad Dermatol Venereol 2020;34(7):e306-e307

17 Hunt M, Koziatek C. A case of COVID -19 pneumonia in a young male with full body rash as a presenting symptom. Clin Pract Cases Emerg Med 2020;4(2):219-221

18 Joob B, Wiwanitkit V. COVID-19 can present with a rash and be mistaken for dengue. J Am Acad Dermatol 2020;82(5):e177

19 Mahé A, Birckel E, Krieger S, Merklen C, Bottlaender L. A distinctive skin rash associated with coronavirus disease 2019? J Eur Acad Dermatol Venereol 2020;34(6):e246-e247

20 Jimenez-Cauhe J, Ortega-Quijano D, Prieto-Barrios M, Moreno-Arrones OM, Fernandez-Nieto D. Reply to "COVID-19 can present with a rash and be mistaken for dengue": Petechial rash in a patient with COVID-19 infection. J Am Acad Dermatol 2020;83(2):e141-e142

21 Ehsani AH, Nasimi M, Bigdelo Z. Pityriasis rosea as a cutaneous manifestation of COVID-19 infection. J Eur Acad Dermatol Venereol 2020. Doi: 10.1111/jdv.16579
22 Amatore F, Macagno $\mathrm{N}$, Mailhe $\mathrm{M}$, et al. SARSCoV-2 infection presenting as a febrile rash. J Eur Acad Dermatol Venereol 2020;34(7):e304-e306

23 Sanchez A, Sohier P, Benghanem S, et al. Digitate papulosquamous eruption associated with severe acute respiratory syndrome coronavirus 2 infection. JAMA Dermatol 2020. Doi: $10.1001 /$ jamadermatol.2020.1704

24 Jones VG, Mills M, Suarez D, et al. COVID-19 and Kawasaki disease: novel virus and novel case. Hosp Pediatr 2020;10(6):537-540

25 Paolino G, Canti V, Mercuri SR. Rovere Querini P, Candiani M, Pasi F. Diffuse cutaneous manifestation in a new mother with COVID-19 (SARS-Cov-2) Int J Dermatol 2020;59(7):874-875

26 Goren A, Vaño-Galván S, Wambier CG, et al. A preliminary observation: Male pattern hair loss among hospitalized COVID-19 patients in Spain - A potential clue to the role of androgens in COVID-19 severity. J Cosmet Dermatol 2020;19(7):1545-1547

27 Lin P, Zhu S, Huang Y, et al. Adverse skin reactions among healthcare workers during the coronavirus disease 2019 outbreak: a survey in Wuhan and its surrounding regions. Br J Dermatol 2020;183(1):190-192

28 Singh M, Pawar M, Bothra A, Choudhary N. Overzealous hand hygiene during the COVID 19 pandemic causing an increased incidence of hand eczema among general population. J Am Acad Dermatol 2020;83(1):e37-e41

29 Magro C, Mulvey JJ, Berlin D, et al. Complement associated microvascular injury and thrombosis in the pathogenesis of severe COVID-19 infection: a report of five cases. Transl Res 2020;220:1-13

30 Xiong M, Liang X, Wei Y-D. Changes in blood coagulation in patients with severe coronavirus disease 2019 (COVID-19): a meta-analysis. Br J Haematol 2020;189(6):1050-1052

31 Zengarini C, Orioni G, Cascavilla A, et al. Histological pattern in COVID-19-induced viral rash. J Eur Acad Dermatol Venereol 2020. Doi: 10.1111/jdv.16569

32 Price KN, Frew JW, Hsiao JL, Shi VY. COVID-19 and immunomodulator/immunosuppressant use in dermatology. J Am Acad Dermatol 2020;82(5):e173-e175

33 Fernandez-Nieto D, Ortega-Quijano D, Segurado-Miravalles G, Pindado-Ortega C, Prieto-Barrios M, Jimenez-Cauhe J. Comment on: cutaneous manifestations in COVID-19: a first perspective. Safety concerns of clinical images and skin biopsies. J Eur Acad Dermatol Venereol 2020;34(6):e252-e254

34 Lim HW, Feldman SR, Van Voorhees AS, Gelfand JM. Recommendations for phototherapy during the COVID-19 pandemic. J Am Acad Dermatol 2020;83(1):287-288

35 Freeman EE, McMahon DE, Fitzgerald ME, et al. The American Academy of Dermatology COVID-19 registry: Crowdsourcing dermatology in the age of COVID-19. J Am Acad Dermatol 2020;83(2):509-510 